\title{
Environmental Correlates of Objectively Measured Physical Activity and Sedentary Behavior in After-School Recreation Sessions
}

\author{
Richard R. Rosenkranz, Greg J. Welk, and David A. Dzewaltowski
}

\begin{abstract}
Background: Active recreation sessions taking place within after-school programs (ASP) present an opportunity for attending children to attain part of the recommended 60 minutes of daily moderate-to-vigorous physical activity (MVPA). This cross-sectional study's purpose was to assess relationships between microlevel ASP environmental characteristics and physical activity and sedentary behavior (SED). Methods: During 161 ASP active recreation sessions, 240 children from 7 schools wore Actigraph GT1M accelerometers and were observed up to 6 times per year, over 3 years. To provide microlevel environmental data, trained observers recorded session times, location, duration, organization, equipment, and number of children and staff. Unadjusted bivariate correlations and multivariable regression analyses were used to assess the influence of microlevel environmental variables on MVPA and SED, with regression models controlling for relevant covariates. Results: Across all ASP active recreation sessions, children spent $39 \pm 15 \%$ in MVPA and $16 \pm 11 \%$ in SED. Session location, boy-to-girl ratio, and duration were significantly related to MVPA in the regression model. For SED, location and duration were significant influences in the model. Conclusions: Both location and duration appear to be modifiable correlates of group physical activity level, which may serve to inform intervention efforts to promote physical activity in ASP.
\end{abstract}

Keywords: children, exercise, accelerometer, determinants

Moderate-to-vigorous-intensity physical activity (MVPA), is associated with numerous positive health-related outcomes in children and adolescents (collectively termed youth), including physical fitness, musculoskeletal health, and the prevention of obesity. ${ }^{1}$ Recent investigations have also focused on academic outcomes associated with MVPA, and have found positive influences from MVPA on concentration, memory, and classroom behavior. ${ }^{2}$ Sedentary behavior (SED), which reflects waking time with little physical movement and low levels of energy expenditure, has been linked with obesity and other negative health-related outcomes in youth. ${ }^{3}$ Often, television viewing or other "screen time" activities are used as a proxy measure for SED, and there is good evidence for an association between hours of watching television or screen time and metabolic syndrome, overweight and obesity in youth. ${ }^{3}$

Many American youth do not meet the current recommendations of 60 minutes MVPA daily ${ }^{4}$ and

Rosenkranz is with the School of Biomedical and Health Sciences, University of Western Sydney, Australia, and the Dept of Human Nutrition, Kansas State University, Manhattan, KS. Welk is with the Dept of Health and Human Performance, Iowa State University, Ames, IA. Dzewaltowski is with the Dept of Kinesiology, Kansas State University, Manhattan, KS. subsequently are at risk for negative health consequences. ${ }^{1}$ Furthermore, the proportion of youth meeting the MVPA guidelines tends to drop from childhood to adolescence. ${ }^{4}$ Similarly, many youth engage in sedentary behaviors such as television watching and other screen time more than the American Academy of Pediatrics' recommended ${ }^{5}$ maximum of 2 hours per day. ${ }^{6}$ Collectively, the abundance of inactive youth represents a populationlevel missed opportunity for health gain gained from an active lifestyle and simultaneously poses a public-health problem with respect to risk for chronic disease. ${ }^{1,3}$

In accordance with the Behavioral Epidemiological Framework, ${ }^{7}$ research focused on investigating correlates or determinants of MVPA and SED is important for the development of evidence-based interventions designed to increase MVPA or decrease SED in youth populations. Knowledge of nonmodifiable correlates, those that are not able to be readily changed, (eg, gender, foul weather) can assist in the selection of target populations or the creation of tailored interventions. ${ }^{8}$ On the other hand, knowledge of modifiable correlates can assist in the development of effective interventions. ${ }^{9}$ Presently there are a number of high-quality literature reviews available on both nonmodifiable and modifiable correlates of MVPA in children and adolescents $8,10,11$ and one of these also addressed correlates of SED. ${ }^{8}$ In the literature, there has been consistent evidence for the following correlates 
of MVPA in children: sex (male), parental physical activity, social support, weight status, physical activity preferences, intention to be active, perceived barriers (inverse), previous physical activity, healthy diet, access to facilities or programs, self efficacy, and time spent outdoors. ${ }^{8,10}$ With regard to SED, the evidence base is presently too small to show consistent findings that can be interpreted with confidence, although there is some evidence for the following correlates of SED in children: ethnicity, single-parent household, and urban versus suburban dwelling. ${ }^{8}$ Unfortunately, many of these studies within the systematic reviews are limited by self-report or proxy measures of MVPA and SED that are prone to various biases, and further work with objective measures will be useful to confirm or refute the previously reported observed associations.

Until recent years, research on correlates of MVPA tended to focus heavily on psychosocial factors. ${ }^{10}$ More recently, correlate research has begun to address broader environmental factors. ${ }^{11-13}$ According to the ANGELO (Analysis Grid for Environments Linked to Obesity) ecological framework, environmental factors can be classified into 4 types: physical, sociocultural, economic, and policies. ${ }^{11}$ Each type of environmental factor can also be conceptually defined, identified, and measured with a microlevel environment (smallest in scope, most proximal to an individual; eg, classroom), or macro-level environment (largest in scope; eg, school district) within environmental settings ranging from the classroom, to the school, neighborhood, city, and so on. ${ }^{11}$

Physical activity researchers have used both subjective self-report data and objective methods such as direct observation or geographic information systems (GIS) to assess potential relationships between microlevel or macro-level environmental attributes and MVPA or SED. ${ }^{11-15}$ In research on youth MVPA, researchers have recently begun to investigate microlevel influences of the school environment on MVPA in youth. ${ }^{16-24}$ Although the evidence base is rapidly growing with respect to microlevel correlates of youth MVPA in the school setting, many studies do not use objective measures of environments, MVPA, or SED, fail to control nuisance variables in the analysis, or do not assess environmental influences when children have apparently equal opportunities to be physically active within a defined behavior setting. ${ }^{11}$

The after-school period appears to be a critical window of opportunity for children to be physically active $^{25}$ and after-school programs are one example of a setting where populations of children may participate in substantial amounts of MVPA during this critical window. ${ }^{26,27}$ To our knowledge, no previous studies have focused specifically on the microlevel environmental correlates of MVPA and SED in after-school program recreation sessions. Therefore, the purpose of this study was to investigate the associations between microlevel environmental characteristics of after-school program active recreation sessions and session-level MVPA and SED among attending youth. Specifically, our aim, based on a public-health focus, was to use active recreation sessions as the unit of analysis to determine the influence of directly observed microlevel after-school environmental variables on objectively measured MVPA and SED. Such an approach lends itself to the development and testing of potential policy-level interventions designed to promote activity in populations of children, based on manipulations to the environmental features of active recreation sessions.

\section{Methods}

This cross-sectional study is based on data analyses of 161 active recreation sessions attended by children $(\mathrm{N}=$ 240 ) in third or fourth grade (ages 8 to 10 years) at 1 of 7 after-school programs in the Healthy Opportunities for Physical Activity and Nutrition (HOP'N) After-School Study. ${ }^{28} \mathrm{HOP}$ ' $\mathrm{N}$ was a group-randomized controlled trial conducted over 3 years that was designed to prevent childhood obesity through increased opportunities for MVPA (at least 30 minutes daily, extra sports/games equipment) and healthful eating, staff training, and an educational curriculum delivered in after-school programs. The microlevel environmental variables were operationally defined as the directly observed active recreation session characteristics: 1) inside or outside location, 2) organized or free play activity, 3 ) nonfixed equipment provided or not, 4) total number of children, 5) ratio of girls to boys, 6) ratio of staff members to children, and 7) duration of sessions. These microlevel environmental variables presently under study were not targeted in the HOP'N intervention, though intervention programs did receive staff training that was based on CATCH guidelines ${ }^{29}$ for organized MVPA, and also received CATCH game equipment (eg, balls, hoops, scarves, cones). Staff training for increasing physical activity opportunities in after-school time emphasized active involvement of all attending children, the use of nonelimination games instead of elimination games, and the minimization of inactive instructional management time (ie, giving directions while children sit or stand in place) during active recreation sessions. Multivariable regression models (discussed below) were used to control for the potential influence of the intervention condition, after-school program site, and study year, such that adjusted analyses would portray the independent relationship between microlevel environmental exposures and the outcomes of MVPA and SED.

The current study extends earlier work led by Cole$\operatorname{man}^{26}$ and $\operatorname{Tros}^{27}$ on the HOP'N project, and uses the recreation session as unit of analysis to assess the associations between microlevel environmental variables and session-level accelerometer-measured MVPA and sedentary behavior in active recreation sessions at both intervention $(n=4)$ and control $(n=3)$ sites. The use of recreation session as the unit of analysis is an important feature of our study in aligning with earlier work by McKenzie and colleagues ${ }^{22-24}$ and our use of an ecological framework with a broad public health focus. With that, 
our study findings are meant to represent a population of after-school program active recreation sessions where children attend, and our findings are meant to generalize across this population of sessions, rather than represent or generalize across children.

\section{Sample}

The current study's data were collected during 161 active recreation sessions from 18 observation days per afterschool program site, spread over 3 academic years. Active recreation sessions lasted an average of 27.5 minutes $(\mathrm{SD}=17.6)$, including an average of 16 children $(\mathrm{SD}=$ 10) per session. The mean age of participating children was 9.3 years $(\mathrm{SD}=0.7)$ at baseline of each study year, and was $51 \%$ males. The sample included third graders (22\%), fourth graders (78\%), non-Hispanic Caucasians $(62 \%)$ minority race/ethnicity (38\%), and $49 \%$ were lower socioeconomic status (eligible for free or reduced lunch). Children assented to participate, and their parents signed a consent form before data collection. At each after-school program site, a new cohort in the fourthgrade group was recruited each of the 3 years, so children were not followed longitudinally across years. This study was approved by the IRB at Kansas State University, and by the Lawrence Unified School District 497's research committee.

\section{Observations}

During after-school program (ASP) time, which lasted about 2 hours each day, a research assistant recorded activities and events of the after-school program's fourthgrade group as discrete sessions (defined as an activity that $51 \%$ or more of children were doing as a group) in a customized log book, making note of start and stop times for each session. As previously described, ${ }^{26,27}$ sessions were categorized as one of the following: 1) Active recreation, 2) Nonactive recreation, 3) Academic time, 4) Enrichment, and 5) Snack. Thus, recreational activities were categorized as active or nonactive. Examples of nonactive recreation sessions included playing board games and reading for fun. Active recreation featured some focus on physical activity, either organized by adult leaders or free play. These active recreation sessions (161 sessions across 3 years) typically were located either on the outside playgrounds or in the school gymnasium.

\section{Environmental Variables}

As previously described, ${ }^{26}$ each after-school active recreation session was evaluated using the environmental support categories from the System for Observing Play and Leisure Activity in Youth (SOPLAY ${ }^{30}$ ). Sessions were coded dichotomously based on whether the environments were 1) usable for physical activity, 2) supervised by after-school personnel, 3) equipped beyond permanent improvements, and 4) organized by adult leaders. All 161 sessions were observed to be usable for physical activity and supervised by adult leaders. Active recreation session organization was dichotomized as either organized or free play. Organized active recreation featured close supervision and direction by an adult leader, with adultdefined rules, boundaries, and expectations. In contrast, free play was remotely supervised by an adult and did not involve adult-defined rules, boundaries, and expectations. With SOPLAY observations, research assistants also recorded the session location (dichotomized as inside or outside), as well as the number of boys, girls, and staff present. From research assistant observations, we derived environmental variables for each session, including duration, number of children, session organization, location, equipment availability, boy-to-girl ratio, and staff-to-student ratio,

\section{Physical Activity and Sedentary Behavior}

As previously described, ${ }^{27}$ physical activity level was measured objectively using Actigraph GT1M uniaxial accelerometer-based monitors (Shalimar, FL), employing a 30-sec epoch. Monitors were emplaced by trained research assistants and worn by participants at the waist (right iliac crest) on an adjustable elastic belt. Teams of 2 research assistants attended each after-school program site 6 days per school year for 3 years to observe and collect physical activity data. Accelerometers were worn on 1 observation day every 4 to 5 weeks, including a Monday, Tuesday, and Thursday (in random order) during each semester, totaling 6 days across both semesters. Participating children wore Actigraph monitors for the entirety of their after-school program attendance each data collection day. Upon children's arrival at the program, research assistants attached an Actigraph monitor to each child's hip (at right iliac crest) using an elastic belt. In a logbook, research assistants noted time of monitor attachment, child participant number, and accelerometer identification number. At the end of the program day, or when the child left the program, a research assistant removed the Actigraph, making note of the time.

Accelerometer count data for each child in each active recreation session were uploaded to a customized computer program to determine the percentage of active recreation session time spent in SED $(<100$ counts per minute $^{27}$ ) and MVPA ( $\geq 4$ metabolic equivalents). The age-specific counts-per-minute threshold for 4 metabolic equivalents was derived from a Freedson and colleagues' MET prediction equation, ${ }^{31}$ divided by 2 to accommodate the $30-$ sec epoch length. Similarly, the 100 counts-perminute threshold was also divided by 2 to accommodate the 30 -sec epoch length. Active recreation session codes and start/stop times, child attendance, and accelerometer wear times were used to ensure only the data of children who attended and wore a monitor in each active recreation session were analyzed. Nonwear time was assessed by counting 20 or more consecutive epochs of zero counts, and these nonwear data were excluded from analyses. 
Thirty minutes of valid accelerometer data per child per after-school program day were required, a priori, for inclusion in analyses, and accelerometer files with less than 30 minutes were excluded from analyses. Means for all attending children within each individual active recreation session were computed to render a sessionlevel percentage of time spent in MVPA and SED for all 161 sessions, and these means were then merged with the session-level observational environmental variable dataset by matching after-school program, date, session number, and start/stop time.

\section{Statistical Analysis}

Descriptive statistical analyses were performed using SPSS (version 18.0; SPSS Inc., Chicago, IL). Inferential statistical analyses were performed using SAS software (version 9.1; SAS Institute, Cary, NC). Microlevel environmental variables used in analyses included dichotomous (session location inside or outside, session equipment present or not, session organized or free play), ratio (boy-to-girl ratio, staff-to-student ratio) and interval variables (session duration in minutes, session number of children). Spearman correlation (for ratio and interval variables) or point-biserial (for dichotomous variables) correlation coefficients were used to assess the unadjusted association between each microlevel environmental variable and percentage of time in MVPA and SED. Associations between environmental variables and MVPA or SED were examined using a linear regression model (PROC MIXED), controlling for study year $(1,2,3)$, intervention condition (intervention, control), and observation number, with after-school program site as random effect (to address clustering of sessions within after-school programs). An alpha level for significance was set at 0.05 .

\section{Results}

Across all 161 active recreation sessions, the mean percentage of time spent in MVPA was $39.0 \%$ (SD = $14.6 \%$ ). Across these 161 active recreation sessions, the mean percentage of time spent in SED was $16.1 \%$ (SD = $10.9 \%$ ). For descriptive purposes, Table 1 displays means and standard deviations of MVPA and SED by session organization, session location, and session equipment.

Across sessions, percent time in MVPA and SED showed a strong inverse correlation $\left(r_{s}=-0.741, P\right.$ $<.001)$. Among the unadjusted bivariate correlation analyses, MVPA was significantly correlated with session organization $\left(r_{p b}=-0.268, P=.001\right)$ and session location $\left(r_{p b}=-0.274, P<.001\right)$. Among the unadjusted bivariate correlation analyses, MVPA was not significantly correlated with session equipment $\left(r_{p b}=0.124, P=.118\right)$, boy-to-girl ratio $\left(r_{s}=0.146, P=.067\right)$, session number of children $\left(r_{s}=.148, P=.062\right)$, session duration $\left(r_{s}=\right.$ $-0.116, P=.147)$, or staff-to-student ratio $\left(r_{s}=-.093\right.$, $P=.247)$.

Among the unadjusted bivariate correlations, SED was significantly correlated with session organization $\left(r_{p b}=0.156, P=.048\right)$, session location $\left(r_{p b}=0.247, P\right.$ $=.002)$ and session duration $\left(r_{s}=0.170, P=.032\right)$. In the unadjusted bivariate correlation analyses, SED was not significantly correlated with session equipment $\left(r_{p b}\right.$ $=-0.123, P=.119)$, boy-to-girl ratio $\left(r_{s}=-0.073, P=\right.$ $.363)$, session number of children $\left(r_{s}=-0.094, P=.237\right)$, or staff-to-student ratio $\left(r_{s}=0.046, P=.564\right)$.

Multiple linear regression analyses (shown in Table 2) were used to examine the associations between microlevel environmental variables and physical activity levels, adjusting for potential covariates. The regression model presented in Table 2 depicts the influence of a 1-unit change in each microlevel environmental variable on

Table 1 Descriptive Statistics of Physical Activity Levels by Session Organization, Location, and Equipment Status

\begin{tabular}{lcc}
\hline & $\begin{array}{c}\text { Mean percent time } \\
\text { in MVPA (SD) }\end{array}$ & $\begin{array}{c}\text { Mean percent time } \\
\text { in SED (SD) }\end{array}$ \\
\hline $\begin{array}{l}\text { All active recreation sessions }(\mathrm{n}=161) \\
\text { Session organization }\end{array}$ & $39.0(14.6)$ & $16.1(10.9)$ \\
$\quad$ Organized $(\mathrm{n}=81)$ & $35.4(16.0)$ & $17.4(10.5)$ \\
$\quad$ Free play $(\mathrm{n}=80)$ & $42.6(12.2)$ & $14.7(11.2)$ \\
Session location & & \\
$\quad$ Inside $(\mathrm{n}=86)$ & $35.5(15.3)$ & $18.5(11.5)$ \\
$\quad$ Outside $(\mathrm{n}=75)$ & $43.0(12.7)$ & $13.2(9.6)$ \\
Session equipment & & $18.4(9.8)$ \\
$\quad$ No equipment provided $(\mathrm{n}=23)$ & $34.2(14.6)$ & $15.7(11.1)$ \\
$\quad$ Equipment provided $(\mathrm{n}=138)$ & $39.8(14.5)$ &
\end{tabular}

Abbreviations: MVPA, moderate-to-vigorous physical activity; SED, sedentary behavior. 
Table 2 Results of Multiple Regression Analyses Examining Microlevel Environmental Variables Contributing to Physical Activity Level Within After-School Program Active Recreation Sessions

\begin{tabular}{lcc}
\hline Model components $^{\mathrm{a}}$ & MVPA & SED \\
\hline Session organized $^{\mathrm{b}}$ & -2.5 & 1.9 \\
Session located inside $^{\mathrm{b}}$ & $-5.4^{*}$ & $5.4^{*}$ \\
Session equipment provided $^{\mathrm{b}}$ & 3.2 & -1.8 \\
Intervention condition $^{\mathrm{b}}$ & 1.2 & -0.9 \\
Boy-to-girl ratio $^{\mathrm{c}}$ & $15.9^{*}$ & -6.3 \\
Staff-to-child ratio $^{\mathrm{c}}$ & -7.7 & -1.7 \\
Session number of children $^{\mathrm{d}}$ & -0.1 & -0.6 \\
Session duration in minutes $^{\mathrm{d}}$ & $-0.2^{*}$ & $0.1^{*}$ \\
Intercept & $43.7^{*}$ & $14.9^{*}$ \\
\hline
\end{tabular}

Abbreviations: MVPA, moderate-to-vigorous physical activity; SED, sedentary behavior.

a Data are unstandardized coefficients, reflecting the amount of change in percentage of time in MVPA or SED for each 1-point change in the variable, controlling for all other model variables, plus afterschool program site, study year, and observation number.

${ }^{\mathrm{b}}$ Dichotomous variable.

${ }^{c}$ Ratio variable.

${ }^{\mathrm{d}}$ Interval variable.

$* P<.05$.

the percentage of time spent in MVPA or SED. In the regression models for MVPA, we assessed the independent contributions of session structure, session location, session duration, session equipment, session number of children, boy-to-girl ratio, and staff-to-student ratio, while controlling for after-school program site, study year, observation number, and intervention condition. In this model, the type III tests of fixed effects showed that session location $\left(F_{1,144}=4.13, P=.044\right)$, session duration $\left(F_{1,144}=6.72, P=.010\right)$, and boy-to-girl ratio $\left(F_{1,144}=4.80, P=.030\right)$ made significant contributions to the MVPA model. In contrast, the type III tests of fixed effects showed that session structure $\left(F_{1,144}=0.84, P=\right.$ $.360)$, session equipment $\left(F_{1,144}=1.08, P=.301\right)$, session number of children $\left(F_{1,144}=0.58, P=.447\right)$, and staffto-student ratio $\left(F_{1,144}=1.77, P=.186\right)$ did not make significant contributions to the MVPA model.

In the regression models for SED, we paralleled the statistical analysis for MVPA by assessing the independent contributions of session structure, session location, session duration, session equipment, session number of children, boy-to-girl ratio, and staff-to-student ratio, while controlling for after-school program site, study year, observation number, and intervention condition. In this SED model, the type III tests of fixed effects showed that session location $\left(F_{1,144}=6.92, P=.009\right)$ and session duration $\left(F_{1,144}=5.63, P=.019\right)$, made significant contributions to the SED model. In contrast, the type III tests of fixed effects showed that session structure $\left(F_{1,144}\right.$ $=0.83, P=.364)$, session equipment $\left(F_{1,144}=0.60, P\right.$ $=.441)$, session number of children $\left(F_{1,144}=0.44, P=\right.$
$.508)$, boy-to-girl ratio $\left(F_{1,144}=1.30, P=.256\right)$ and staffto-student ratio $\left(F_{1,144}=0.15, P=.698\right)$ did not make significant contributions to the SED model.

\section{Discussion}

Our study sought to investigate the associations between microlevel environmental characteristics of after-school program active recreation sessions and session-level MVPA and SED of attending youth. Across 18 observation days over 3 academic years at 7 program sites, we observed 161 active recreation sessions, about 1.3 per day. Active recreation sessions were homogeneous with regard to supervision and usability for physical activity, as all sessions were supervised by at least 1 adult leader, and all were located in school areas that were usable for physical activity. Active recreation sessions were heterogeneous with regard to number of attending children, staff-to-student ratio, boy-to-girl ratio, session organization, session location, session duration, and presence of equipment for physical activity. On average, these active recreation sessions engaged attending children in MVPA for $39 \%$ of session time, and children were sedentary for an average of $16 \%$ of active recreation session time. Thus, a given active recreation session at an after-school program in our study contributed about 11 minutes of MVPA (totaling about 14 minutes per after-school program day from active recreation sessions) toward a recommended total daily accumulation of 60 minutes per day for schoolaged youth. ${ }^{1}$ This study's total is lower than the findings of Trost and colleagues, ${ }^{27}$ but the present paper focused 
only on the contribution of active recreation sessions, rather than MVPA attained in all types of sessions during after-school program time.

The main finding of our study was that in the adjusted regression models, the microlevel environmental characteristics of the active recreation sessions were significantly associated with both the percentage of time spent in MVPA (specifically session location, session duration, boy-to-girl ratio) and also SED (specifically session location, session duration). Although research on after-school program activity levels is limited, comparisons of the current findings to earlier school-based research on school recess and physical education may provide interesting insights, especially given that we observed a nearly even mix of structured active recreation sessions (similar to structured physical education classes) and free-play active recreation sessions (similar to recess). Aside from previous studies using HOP' $N$ after-school first-year data, ${ }^{26,27}$ this finding supports a previous school-based study of middle-school youth that showed environmental characteristics explained $42 \%$ of the variance in the proportion of girls who engaged in MVPA, and $59 \%$ of the variance for boys. ${ }^{18}$ In particular, in the current study, sessions held outdoors were significantly more physically active, and also less sedentary, compared with sessions held indoors. This aligns with McKenzie's ${ }^{22}$ observational study of 293 third-grade physical education classes, where lower percentages of time spent sitting and higher percentages of time spent walking, being very active and engaged in MVPA were seen in the outdoor classes. Our findings also agree with Trost's ${ }^{21}$ finding for MVPA, but simultaneously controls for session organization, and extends the work to show an association between location and SED. Unlike the earlier findings from HOP' $N,{ }^{26,27}$ our results did not find a significant association for session organization, in that there was no significant difference between free play and organized sessions when controlling for all covariates in the adjusted regression model. Outdoor active recreation sessions were most likely to be free play instead of organized, and the organized sessions were most likely to occur inside, so it appears that when controlling for indoor versus outdoor location, the influence of session organization became nonsignificant.

Our finding that youth were more active and less sedentary outdoors indirectly supports 2 previous studies on school recess ${ }^{17}$ and preschool recess ${ }^{16}$ that both found less space per child was associated with lower activity levels. Although our study was limited in that we did not directly measure space per child, all of the outdoor playground areas provided more space per child than the indoor playground areas available at each after-school program site. It is also possible that outdoor activity levels were greater than indoor activity levels for reasons beyond just the available space, such as differing recreational or instructional objectives of staff. ${ }^{22}$ Furthermore, each outdoor after-school program site was equipped with playground structures such as slides, swings, climbing towers, and other fixed equipment, which could have impacted activity levels. Our observed variation in MVPA and SED by location finding also corresponds more generally with previous research showing that time spent outside is associated with greater levels of free-living or habitual MVPA in youth. ${ }^{10}$ The present finding is novel, in that no previous study has investigated whether active recreation sessions in after-school programs differ in SED levels by indoor versus outdoor location, and few have investigated this location issue for MVPA. ${ }^{27}$

In terms of actual effect size, our finding of MVPA and SED variability by location indicates that children were likely to accumulate about 1.5 more minutes of MVPA, and about 1.5 minutes less of SED in an averageduration active recreation session held outdoors, versus indoors. For children attending a program featuring 1 active recreation session per day, this difference between outdoors and indoors could theoretically add up to an additional 1 full hour of MVPA, and also 1 full hour less of SED every 8 weeks. This influence on physical activity levels could be meaningful at the population level in terms of energy balance and health-related outcomes.

Among the most robust of findings in the correlate literature for youth physical activity is that boys tend to be more physically active than girls. ${ }^{8,10}$ It is not surprising then that our study showed sessions had higher levels of MVPA commensurate with higher boy-to-girl ratios of attending children, even when controlling for other covariates, but we found no significant relationship between boy-to-girl ratio and SED. Although our study was framed around environmental session-level variables rather than individual-level variables, related research from HOP' $N$ has shown that girls are less active than boys in after-school time. ${ }^{27,32}$ Research by McKenzie and colleagues has also shown that boys tend to be more active than girls in physical education classes, and particularly during free play activity. ${ }^{22,23}$ Research in the school recess context also has shown this sex difference. ${ }^{19,20}$ Our study adds to the burgeoning area of literature showing that girls frequently attain lower levels of MVPA, even when having apparently equivalent environmental settings and opportunities for physical activity. ${ }^{16,17,19,22,32}$ Future studies in this area should examine whether sex moderates the relationships between microlevel environmental variables and physical activity levels of children, as it is possible that boys and girls may be differentially more active or sedentary in response to certain environmental features.

Our findings also indicated that the duration of active recreation sessions was influential on the level of physical activity for attending children. Generally, the longer the active recreation session, the lower the percentage of time spent in MVPA and greater the percentage of time spent in SED. With regard to actual effect size of session duration in the current study, for every 5 minutes of active recreation session duration, there was a 1 percentage point drop in MVPA, and for every 10 minutes, there was a 1 percentage point increase in SED. Presumably, children may have gradually lost interest or become physically fatigued as active recreation sessions continued, but the current study did not measure such explanatory variables. This inverse relationship between session duration and 
activity level is similar to Cardon's study ${ }^{16}$ of preschool children in a recess context, and also to Chow's study ${ }^{24}$ of school-aged children in physical education classes, which found a weak inverse association $(r=-0.13)$ in sessions that lasted about 32 minutes - similar to our study's sessions. In contrast, Zask and colleagues ${ }^{19}$ indirectly investigated the impact of recess duration, and found higher activity levels during 30-minute lunch-time recess periods, in comparison with 16-minute morning recess periods. Of note, however, the lunch and morning recesses in Zask's study likely differed in other important ways, beyond just differing in duration. ${ }^{19}$ Guinhoya and colleagues ${ }^{21}$ experimentally investigated the activity levels of children by alternating the duration of recess between 15 and 20 minutes. These authors found greater absolute amounts of MVPA with longer recess periods, but there were similar percentages of time spent in MVPA. ${ }^{21}$ Lastly, Ridgers and colleagues ${ }^{20}$ investigated the use of playground markings on activity levels of school-aged youth, and found that recess duration was not a significant predictor of MVPA, though the intervention appeared to be more effective with longer recess periods. Thus, further research is required to provide more clear answers about the relationship between session or class duration and activity levels, and it is possible that the relationship is not a linear one. Future studies should seek to flesh out the relationship between duration and physical activity levels in varying physical activity contexts, and to conduct randomized controlled trials with manipulations of session duration as an independent variable, and to investigate potential mechanisms of decline in physical activity over time.

With regard to provision of equipment, we did not find that the presence of physical activity equipment was influential on MVPA or SED in either unadjusted correlations, or in models controlling for other environmental variables and covariates. This finding corresponds to the work of Zask and colleagues, ${ }^{19}$ who found that equipment in general (fixed equipment, balls, other nonfixed equipment) was not significantly related to MVPA, but found there was a positive relationship between the number of balls available and vigorous physical activity. In the current study, we coded sessions as being equipped, based on balls or other nonfixed equipment being available and accessible to the children. Children in our study had access to what Sallis and colleagues ${ }^{18}$ termed "improvements" in the form of permanent playground equipment (fixed equipment) and gymnasiums with floor markings which could have resulted in no additional impact on physical activity from nonfixed equipment such as balls, discs, hoops, jump-ropes, or similar items.

The present findings should be viewed within the context of the inherent limitations of a cross-sectional study. With this study design, we can determine only a level of association between microlevel environmental variables and session-level MVPA or SED, and there could potentially be important confounding variables that were not accounted for in this study. The lack of a temporal element and the lack of manipulation of environmental variables in the study serve to discourage any causal inference. Future studies could use prospective cohort or experimental study designs to enable potential causal inferences regarding environmental characteristics and MVPA or SED. Counterbalancing these limitations are several strengths. First, we used rigorous objective measures of MVPA and SED, and carefully matched the accelerometer-derived data with environmental data. Second, these microlevel environmental data were obtained through a labor-intensive process of direct observation, using trained research assistants and validated instruments for data collection. Third, we measured all active recreation sessions over 6 observation days per academic year, for 3 years at 7 sites. With so many observations spanning the full academic year, we believe that our data offer a reliable estimate of physical activity levels in these after-school programs, and bias from seasonality is unlikely. Lastly, our data analysis procedures added to the study's strength in that they allowed for both an unadjusted assessment of association, as well as a fully adjusted model that accounted for important covariates in determining the unique influence of each microlevel environmental variable on MVPA and SED. This comprehensive view of the environmental correlates, with and without adjusting for potential confounders, should help practitioners and researchers to identify appropriate variables for future interventions and to understand key influences on physical activity and sedentary behavior in active recreation sessions.

In conclusion, our study showed that the observed after-school programs provided about 1.3 active recreation sessions per day, each lasting an average about 28 minutes. For every 10 minutes of active recreation offered, roughly 4 minutes were spent in MVPA, and the activity levels of attending children could potentially be increased by holding sessions outside instead of holding sessions inside. Longer active recreation sessions were associated with lower activity levels. So, all else being equal, multiple shorter sessions may be preferable to 1 long session, but this may be logistically difficult for after-school program staff to implement in terms of additional child management or transition time from one after-school area or activity to another. Using microlevel environmental correlate study findings such as these, public health intervention efforts targeting after-school programs may assist children in reducing sedentary time and meeting the guideline of 60 minutes of MVPA daily.

\section{Acknowledgments}

We thank our team of undergraduate research assistants, plus Karla Foster, Karly Geller, David Goodrich, Tanis Hastmann and Sara Rosenkranz for assisting with data collection and management. We thank the people of USD 497 and Boys and Girls Club in Lawrence, KS for their participation and assistance. 
This study was supported by NRI Grant \#2005-35215-15418 from the USDA Cooperative State Research, Education, and Extension Service Human Nutrition and Obesity Program. The HOP' $N$ trial is registered at www.clinicaltrials.gov (No: NCT01015599).

\section{References}

1. Physical Activity Guidelines Advisory Committee. Physical activity guidelines advisory committee report, 2008. Washington, DC: US Department of Health and Human Services; 2008.

2. Trudeau F, Shephard RJ. Physical education, school physical activity, school sports and academic performance. Int J Behav Nutr Phys Act. 2008;5:10.

3. Tremblay MS, Colley RC, Saunders TJ, Healy GN, Owen N. Physiological and health implications of a sedentary lifestyle. Appl Physiol Nutr Metab. 2010;35(6):725-740.

4. Troiano RP, Berrigan D, Dodd KW, et al. Physical activity in the United States measured by accelerometer. Med Sci Sports Exerc. 2008;40(1):181-188.

5. Krebs NF, Jacobson MS, American Academy of Pediatrics Committee on Nutrition. Prevention of pediatric overweight and obesity. Pediatrics. 2003;112(2):424-430.

6. Kaiser Family Foundation. Generation M: media in the lives of 8-18 year olds report. Menlo Park, CA, Henry J. Kaiser Family Foundation; 2005. Available at: http://www.kff.org.

7. Sallis J, Owen N, Fotheringham M. Behavioral epidemiology: a systematic framework to classify phases of research on health promotion and disease prevention. Ann Behav Med. 2000;22(4):294-298.

8. van der Horst K, Paw MJ, Twisk JWR, et al. A brief review on correlates of physical activity and sedentariness in youth. Med Sci Sports Exerc. 2007;39(8):1241-1250.

9. Baranowski T, Lin LS, Wetter DW, Resnikow K, Hearn MD. Theory as mediating variables: why aren't community interventions working as desired? Ann Epidemiol. 1997;S7:S89-S95.

10. Sallis JF, Prochaska JJ, Taylor WC. A review of correlates of physical activity of children and adolescents. Med Sci Sports Exerc. 2000;32(5):963-975.

11. Ferreira I, van der Horst K, Wendel-Vos W, Kremers $\mathrm{S}$, van Lenthe FJ, Brug J. Environmental correlates of physical activity in youth-a review and update. Obes Rev. 2006;8:129-154.

12. Brownson RC, Baker EA, Housemann RA, Brennan LK, Bacak SJ. Environmental and policy determinants of physical activity in the United States. Am J Public Health. 2001;91(12):1995-2003.

13. Crawford D, Cleland V, Timperio A, et al. The longitudinal influence of home and neighbourhood environments on children's body mass index and physical activity over 5 years: the CLAN study. Int J Obes. 2010;34;1177-1187.

14. Swinburn B, Egger G, Raza F. Dissecting obesogenic environments: the development and application of a framework for identifying and prioritizing environmental interventions for obesity. Prev Med. 1999;29:563-570.

15. Duncan M, Mummery K. Psychosocial and environmental factors associated with physical activity among city dwellers in regional Queensland. Prev Med. 2005;40(4):363372.
16. Cardon G, Van Cauwenberghe E, Labarque V, Haerens L, De Bourdeaudhuij I. The contribution of playground factors in explaining children's physical activity during recess. Int J Behav Nutr Phys Act. 2008;5:11.

17. Ridgers ND, Fairclough SJ, Stratton G. Variables associated with children's physical activity levels during recess: The A-CLASS project. Int J Behav Nutr Phys Act. 2010;7:74.

18. Sallis JF, Conway TL, Prochaska JJ, McKenzie TL, Marshall SJ, Brown M. The association of school environments with youth physical activity. Am J Public Health. 2001;91:618-620.

19. Zask A, van Beurden E, Barnett L, Brooks LO, Dietrich UC. Active school playgrounds-myth or reality? Results of "Move It Groove It" project. Prev Med. 2001;33:402408

20. Ridgers ND, Stratton G, Fairclough SJ, Twisk JWR. Children's physical activity levels during school recess: a quasi-experimental intervention study. Int J Behav Nutr Phys Act. 2007;4:19.

21. Guinhouya CB, Hubert H, Dupont G, Durocher A. The recess period: a key moment of prepubescent children's daily physical activity? Electr J Health Educ. 2005;8:126134.

22. McKenzie TL, Feldman H, Woods SE, et al. Children's activity levels and lesson context during third-grade physical education. Res Q Exerc Sport. 1995;66:184-193.

23. McKenzie TL, Marshall SJ, Sallis JF, Conway TL. Student activity levels, lesson context, and teacher behavior during middle school physical education. Res $Q$ Exerc Sport. 2000;71:249-259.

24. Chow BC, McKenzie TL, Lobo L. Children's physical activity and environmental influences during elementary school physical education. J Teach Phys Educ. 2008;27:38-50.

25. Mota J, Santos P, Guerra S, Ribeiro J, Duarte J. Patterns of daily physical activity during school days in children and adolescents. Am J Hum Biol. 2003;15:547-553.

26. Coleman KJ, Geller KS, Rosenkranz RR, Dzewaltowski DA. Physical activity and healthy eating in the after school environment. J Sch Health. 2008;78(12):633-640.

27. Trost SG, Rosenkranz RR, Dzewaltowski DA. Physical activity levels among children attending after-school programs. Med Sci Sports Exerc. 2008;40(4):622-629.

28. Dzewaltowski DA, Rosenkranz RR, Geller KS, et al. HOP'N After-School Project: an obesity prevention randomized controlled trial. Int J Behav Nutr Phys Act. 2010;7:90.

29. Kelder SH, Mitchell PD, McKenzie TL, et al. Long-term implementation of the CATCH physical education program. Health Educ Behav. 2003;30(4):463-475.

30. McKenzie TL, Marshall SJ, Sallis JF, Conway TL. Leisuretime PA in school environments: an observational study using SOPLAY. Prev Med. 2000;30(1):70-77.

31. Freedson P, Pober D, Janz KF. Calibration of accelerometer output for children. Med Sci Sports Exerc. 2005;37(11, Suppl):S523-S530.

32. Rosenkranz RR, Welk GJ, Hastmann TJ, Dzewaltowski DA. Psychosocial and demographic correlates of objectively measured physical activity in structured and unstructured after-school recreation sessions. J Sci Med Sport. 2011;14(4):306-311. 